\title{
Спектр двойных акцепторов в слоях барьеров и квантовых ям гетероструктур $\mathrm{HgTe} / \mathrm{CdHgTe}$
}

\author{
(C) Д.В. Козлов ${ }^{1,2}$, В.В. Румянцев ${ }^{1,2}$, С.В. Морозов ${ }^{1,2}$ \\ ${ }^{1}$ Институт фризики микроструктур Российской академии наук, \\ 603950 Нижний Новгород, Россия \\ ${ }^{2}$ Нижегородской государственный университет им. Лобачевского, \\ 603950 Нижний Новгород, Россия \\ E-mail: dvkoz@ipmras.ru
}

Поступила в Редакцию 24 апреля 2019 г.

В окончательной редакции 29 апреля 2019 г.

Принята к публикации 29 апреля 2019 г.

\begin{abstract}
Проведено теоретическое исследование состояний двойных акцепторов в гетероструктурах $\mathrm{HgTe} / \mathrm{CdHgTe}$ с квантовыми ямами, учитывающее существенную разницу значений диэлектрической проницаемости слоев барьеров и квантовых ям. Влияние такой разницы и возникающего из-за нее наведенного на гетерограницах заряда описывалось с помощью потенциала зарядов-изображений. Расчет показал существенное изменение энергии связи акцепторных центров - вакансий ртути из-за наведенного заряда, при этом энергии ионизации вакансий ртути хорошо согласуются с положением спектральных особенностей в спектре фотолюминесценции гетероструктур $\mathrm{HgTe} / \mathrm{CdHgTe}$ с квантовыми ямами.
\end{abstract}

Ключевые слова: гетероструктуры, двойные акцепторы, дефекты, вакансии ртути, фотолюминесценция, КРТ структуры.

DOI: $10.21883 /$ FTP.2019.09.48128.11

\section{1. Введение}

Уже давно наблюдается интерес к терагерцовому излучению, возникающему за счет переходов с участием мелких примесей в полупроводниках и полупроводниковых структурах с квантовыми ямами (КЯ) [1,2]. Такие структуры являются перспективным материалом для создания источников терагерцового излучения, работающих на оптических переходах между примесными состояниями. До недавнего времени исследования примесей и дефектов проводились в основном в наиболее распространенных и используемых полупроводниковых гетероструктурах на основе таких материалов, как $\mathrm{Ge}$, $\mathrm{GaAs}$ [3], Si [4]. Данная работа является продолжением серии исследований примесно-дефектных центров в гетероструктурах и посвящена расчетам спектра акцепторов в гетероструктурах $\mathrm{HgTe} / \mathrm{CdHgTe} \mathrm{с} \mathrm{квантовыми}$ ямами. Самым распространенным акцептором для таких материалов оказывается вакансия ртути. Она является двухвалентным акцептором и может находиться в трех зарядовых состояниях: нейтральный $A^{0}$-центр, с которым связаны две дырки, $A^{-1}$-центр, с которым связана одна дырка, и не имеющий дырок полностью ионизованный $A^{-2}$-центр. Из-за таких вакансий возникают особенности в спектрах левее полосы межзонных переходов, появляющиеся из-за переходов носителей из валентной зоны на локализованные состояния акцепторов в запрещенной зоне. Фотопроводимость (ФП), связанная с переходами с участием акцепторных состояний, возникает благодаря переходам дырок с акцепторных центров в валентную зону. При этом в формировании такого сигнала $A^{-2}$-центры не участвуют (так как на таких центрах нет дырок), а из-за переходов дырок с состояний $A^{0}$ - и $A^{-1}$-центров в валентную зону в спектре ФП возникают линии, положение которых соответствует энергиям ионизации таких центров (обозначим эти энергии $E_{1}$ и $E_{2}$ соответственно). Фотолюминесценция (ФЛ), связанная с акцепторными центрами, возникает (примеснодефектная ФЛ), напротив, благодаря захвату дырок из валентной зоны на акцепторные центры. При этом в формировании сигнала не участвует полностью заполненный дырками нейтральный $A^{0}$-центр, а при захвате носителей на $A^{-1}$ - и $A^{-2}$-центры испускаются фотоны с энергией, равной соответственно энергии ионизации $A^{0}$-центра $\left(E_{1}\right)$ и $A^{-1}$-центра $\left(E_{2}\right)$. Примесно-дефектная ФЛ наблюдалась как в объемных КРТ (кадмий-ртутьтеллур) пленках, так и в $\mathrm{HgTe} / \mathrm{CdHgTe} \mathrm{c} \mathrm{КЯ} \mathrm{[5].} \mathrm{Для}$ интерпретации наблюдаемых спектров ФЛ и ФП проводились расчеты энергий ионизации вакансий ртути $A^{0}$-центров $\left(E_{1}\right)$ и $A^{-1}$-центров $\left(E_{2}\right)$ [5-7]. Так, в работе [5] проведен расчет спектра двухвалентных акцепторов в объемных КРТ пленках. В работах [6,7] рассчитывался спектр состояний вакансии ртути в $\mathrm{HgTe} / \mathrm{CdHgTe}$ с КЯ при различных положениях иона акцептора в структуре. В этих работах диэлектрическая проницаемость полагалась однородной по всей гетероструктуре. Однако диэлектрические проницаемости слоев барьеров типичных исследуемых гетероструктур (например, $\mathrm{Hg}_{0.3} \mathrm{Cd}_{0.7}$ Те в [7]) и квантовых ям (HgTe) существенно отличаются (составляют соответственно 12.5 и 20.8). Из-за этого на гетерогранице возникает существенный наведенный заряд, который оказывает заметное влияние на потенциал, действующий на носители. В настоящей работе проведен теоретический расчет состояний ва- 
кансий ртути в гетероструктурах $\mathrm{HgTe} / \mathrm{CdHgTe}$ с КЯ, учитывающий такой наведенный заряд.

\section{2. Расчет состояний вакансий ртути в гетероструктурах $\mathrm{HgTe} / \mathrm{CdHgTe}$ с квантовыми ямами}

Были рассчитаны энергии состояний одной (одночастичные состояния) и двух (двухчастичное состояние) дырок, связанных на вакансии ртути при различных расположениях дефекта в гетероструктуре, т.е. найдены энергии отрыва первой и второй дырок от такой вакансии (энергии ионизации $A^{0}$ - и $A^{-1}$-центров). Расчет проводился $k$-p-методом. Гамильтониан уравнения для огибающих волновых функций дырок записывался в виде суммы пяти слагаемых: кинетической энергии (гамильтониан Латтинжера), энергии кулоновского взаимодействия с заряженным акцептором, энергии кулоновского взаимодействия с наведенными зарядами на границе слоев барьеров и КЯ, ограничивающего потенциала квантовой ямы и дополнительного отталкивающего потенциала, существенного вблизи акцепторного центра и описывающего потенциал центральной ячейки (ПЦЯ) [7,8]. При расчетах использовалось аксиальное приближение, т.е. не учитывалась анизотропия закона дисперсии дырок в плоскости, квантовой ямы. Для этого в элементах гамильтониана Латтинжера опущены слагаемые, ответственные за такую анизотропию [6]. Значения параметров Латтинжера и диэлектрической проницаемости в твердом растворе находились линейной интерполяцией между соответствующими значениями параметров в $\mathrm{HgTe}$ и CdTe, взятых из работы [9].

Потенциал наведенных на границе слоев зарядов, возникающий из-за разной диэлектрической проницаемости слоев гетероструктуры, описывают с помощью зарядов изображений. На рис. 1 показана система зарядов, создающая электростатическое поле в двух полубесконечных средах с разными диэлектрическими проницаемостями. На рис. 1, $a$, представляющем заряды, создающие поле в области I (т.е. в среде, в которую помещен внешний заряд), показан внешний заряд (черный кружок) и зарядизображение (серый кружок). Заряд-изображение расположен симметрично внешнему заряду относительно границы раздела сред, и его величина равна

$$
q\left(\varepsilon_{1}-\varepsilon_{2}\right) /\left(\varepsilon_{1}+\varepsilon_{2}\right)
$$

где $q$ - величина внешнего заряда, $\varepsilon_{1}$ и $\varepsilon_{2}$ - диэлектрические проницаемости сред. На рис. $1, b$ показаны заряды, создающие поле в области II. Следует отметить, что положения внешнего заряда и заряда-изображения, создающего поле в этой области, совпадают. В результате оказывается, что в области II электрическое поле создается эффективным зарядом величиной

$$
2 q \varepsilon_{1} /\left(\varepsilon_{1}+\varepsilon_{2}\right) \text {. }
$$
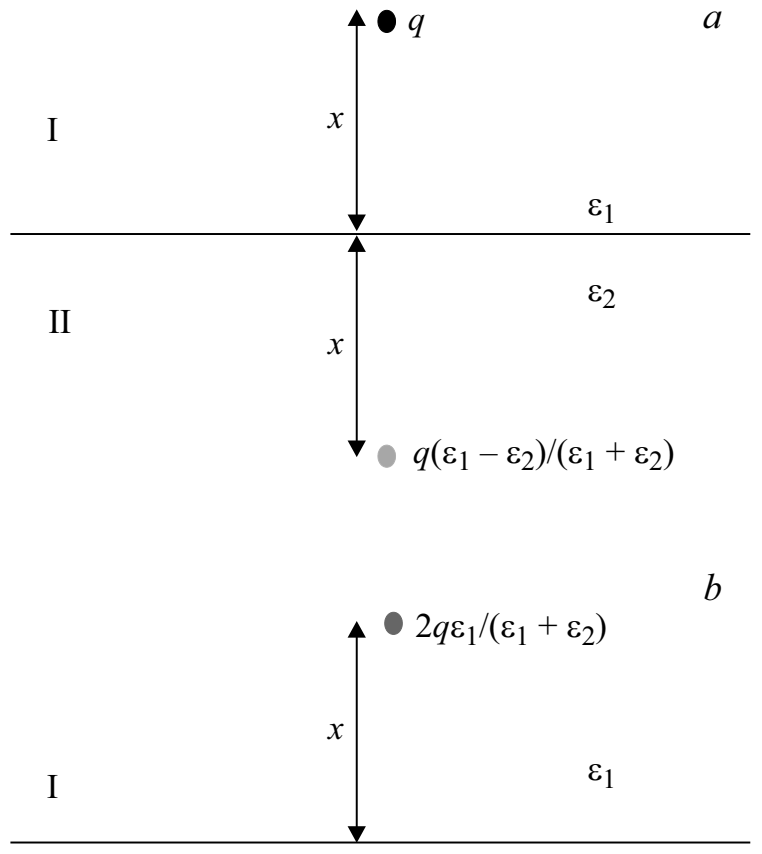

II

$\varepsilon_{2}$

Рис. 1. Схема, показывающая заряды, создающие электростатическое поле в двух полубесконечных средах с разными диэлектрическими проницаемостями: $a$ - заряды, создающие поле в области I; $b$ - представлены заряды, создающие поле в области II. Черным показан внешний заряд, серым заряд-изображение, темно-серым - „эффективный“ заряд на рис. $1, b, x$ - расстояние от внешнего заряда до раздела сред. Отмечены величины зарядов.

Рассмотрим систему зарядов-изображений, создающих электрическое поле в слое с диэлектрической проницаемостью $\varepsilon_{1}$, разделяющем полубесконечные среды с диэлектрической проницаемостью $\varepsilon_{2}$. Такая модель описывает электростатический потенциал, возникающий при внесении иона примеси в структуру $\mathrm{HgTe} / \mathrm{Cd}_{0.7} \mathrm{Hg}_{0.3} \mathrm{Te}$, состоящей из квантовой ямы (в слое $\mathrm{HgTe}$ ) шириной $48 \AA$, окруженной толстыми, толщиной $300 \AA$, барьерами (слоями $\left.\mathrm{Cd}_{0.7} \mathrm{Hg}_{0.3} \mathrm{Te}\right)$. На рис. 2 показаны внешний заряд и заряды-изображения, создающие электрические поля в слое КЯ такой гетероструктуры (в слое с большей диэлектрической проницаемостью $\left.\varepsilon_{1}\right)$ при помещении внешнего заряда в слой КЯ (рис. 2,a) и в слой барьера (рис. 2,b). Видно, что при любомрасположении внешнего заряда возникает последовательность зарядовизображений. Возникает такая последовательность следующим образом:

„Заряды-изображения“ возникают для каждой из двух гетерограниц.

„Заряд-изображение“ для одной первой границы является внешним зарядом для второй границы и для нее возникает изображение такого заряда (изображение изображения), которое в свою очередь оказывается уже внешним зарядом для первой. 


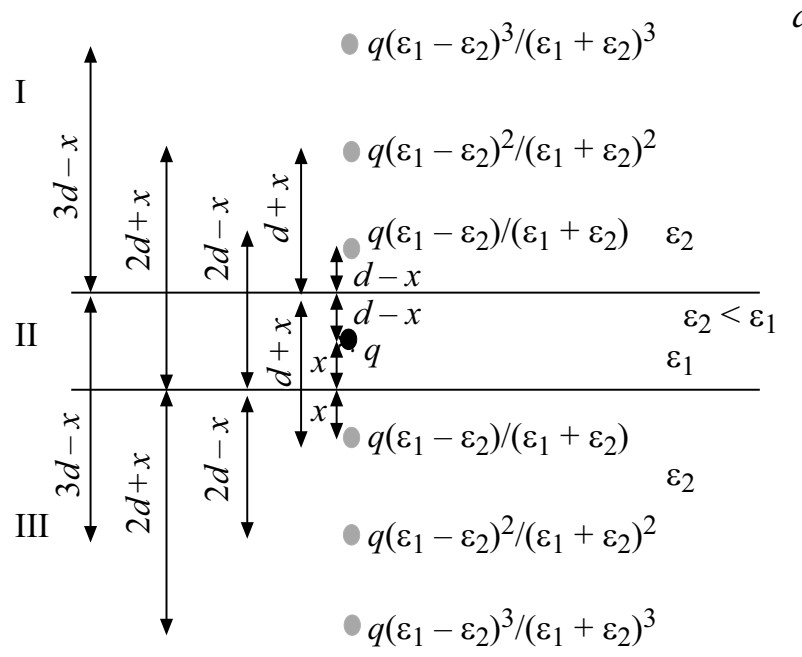

$b$

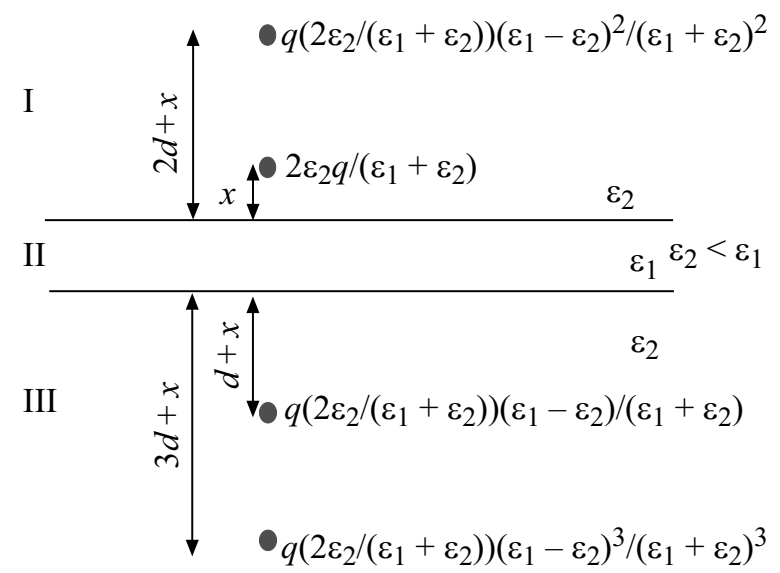

Рис. 2. Схема, показывающая заряды, создающие электрическое поле в слое с диэлектрической проницаемостью $\varepsilon_{1}$ (в области II), разделяющем полубесконечные среды с диэлектрической проницаемостью $\varepsilon_{2} . a-$ внешний заряд находится в разделяющем слое (в слое КЯ); $b-$ внешний заряд помешен в окружающие слои (в слои барьеров). Серым показаны зарядыизображения, черным - внешний заряд, темно-серым эффективные заряды на рис. 2,b. $d$ - ширина КЯ (слоя с диэлектрической проницаемостью $\varepsilon_{1}, x$-расстояние от внешнего заряда до раздела сред.

При каждом шаге такие изображения удаляются от квантовой ямы на расстояние, равное ее ширине, и их заряд уменьшается в $\left(\varepsilon_{1}-\varepsilon_{2}\right) /\left(\varepsilon_{1}+\varepsilon_{2}\right)$ раз (см. рис. 2$)$.

Как было отмечено выше, в гамильтониан для огибающих волновых функций был добавлен еще кулоновский потенциал описанных зарядов-изображений. Отметим, что при расчете состояний акцепторов последовательность зарядов-изображений (см. рис. 2) была оборвана. Заряды-изображения, удаленные от слоя КЯ на расстояние, значительно превышающее масштаб локализации волновой функции основного состояния акцепторного центра (30 § [3]), не учитывались.
Уравнение для огибающих волновых функций дырок с потенциалом акцепторного иона и описанным выше потенциалом зарядов-изображений решали раскладывая волновую функцию акцепторного центра по базису из огибающих волновых функций свободных дырок в квантовой яме $v_{n}^{s}(\mathbf{k}, z) e^{i \mathbf{k} \boldsymbol{\rho}}$, которые являются собственными функциями гамильтониана в отсутствие кулоновского потенциала:

$$
F^{s}(\mathbf{r})=\sum_{n} \int d \mathbf{k} C_{n}(\mathbf{k}) v_{n}^{s}(\mathbf{k}, z) e^{i \mathbf{k} \boldsymbol{\rho}},
$$

где $n$ - номер подзоны размерного квантования, $\mathbf{r}-$ радиус-вектор, $\boldsymbol{\rho}$ - радиус-вектор в плоскости квантовой ямы, $\mathbf{k}$ - волновой вектор дырки. В аксиальном приближении зависимость коэффициентов разложения $C_{n}(\mathbf{k})$ от направления волнового вектора, характеризуемого углом $\alpha$, имеет вид

$$
C_{J}(\mathbf{k}, n, s)=C_{J}(k, n, s) \exp \left[i \alpha\left(J-\frac{3}{2}\right)\right] .
$$

Подставляя разложение (3) в уравнение эффективной массы, получаем интегральное уравнение для коэффициентов разложения. Далее интеграл может быть заменен дискретной суммой по $k$; при этом шаг по $k$ необходимо выбирать меньше обратного боровского радиуса, чтобы подынтегральная функция мало менялась на одном шаге. Суммирование может быть оборвано при $k$, много больших обратного боровского радиуса. Таким образом, задача сводится к диагонализации конечномерной эрмитовой матрицы.

Так были рассчитаны одночастичные состояния дырок на акцепторных центрах и найдены энергии отрыва второй дырки от двойного (двухзарядного) акцептора, т. е. определена энергия связи $A^{-1}$-центра.

Для определения энергии ионизации $A^{0}$-центра необходимо найти энергию состояния двух дырок на вакансии ртути, в котором обе частицы находятся на нижнем энергетическом уровне. Для такого расчета был использован метод самосогласованного поля Хартри [10]. Рассматривалось состояние частицы в поле примесного центра и другой частицы, при этом решались одночастичные уравнение Шредингера для каждой из двух дырок:

$$
\begin{aligned}
& {\left[H_{L}\left(\nabla_{1}\right)+V\left(z_{1}\right)\right.} \\
& \left.\quad-\left(E+U\left(r_{1}\right)-\frac{e^{2}}{2 \kappa} \int \frac{\left|\Psi_{2}\left(r_{2}\right)\right|^{2} d^{3} r_{2}}{\left|r_{1}-r_{2}\right|}\right)\right] \Psi_{1}\left(r_{1}\right)=0, \\
& {\left[H_{L}\left(\nabla_{2}\right)+V\left(z_{2}\right)\right.} \\
& \left.\quad-\left(E+U\left(r_{2}\right)-\frac{e^{2}}{2 \kappa} \int \frac{\left|\Psi_{1}\left(r_{1}\right)\right|^{2} d^{3} r_{2}}{\left|r_{1}-r_{2}\right|}\right)\right] \Psi_{2}\left(r_{2}\right)=0,
\end{aligned}
$$

где $E$ - энергия дырок, $\Psi_{1,2}\left(r_{1,2}\right)-$ волновые функции первой и второй дырок соответственно, $H_{L}\left(\nabla_{1,2}\right)-$ 
гамильтониан Латтинжера соответственно для первой и второй дырок, являющийся матрицей $4 \times 4, V\left(z_{1,2}\right)-$ ограничивающий потенциал квантовой ямы для первой и второй дырок соответственно. $U\left(r_{1,2}\right)$ - суммарная потенциальная энергия первой и второй дырок соответственно в поле иона акцептора, зарядов-изображений и потенциала центральной ячейки. Представляя каждую волновую функцию в виде разложения (3) и подставляя эти разложения в систему (5), получим интегральные уравнения для коэффициентов разложения, которые решали методом последовательных приближений, описанным подробно в работе [6].

\section{3. Результаты и обсуждение}

На рис. 3 представлены рассчитанные зависимости энергии ионизации нейтральной и однократно ионизованной вакансии ртути $\left(A^{0}\right.$ - и $A^{-1}$-центры соответственно) от положения такой вакансии в гетероструктуре $\mathrm{HgTe} / \mathrm{Cd}_{0.7} \mathrm{Hg}_{0.3} \mathrm{Te}$ с КЯ шириной $48 \AA$. Черные линии показывают энергии связи нейтральной вакансии, рассчитанные как без учета влияния наведенных на гетерогранице зарядов (пунктирная линия), так и с учетом влияния зарядов-изображений. Серая линия на рис. 3 показывает энергию связи $A^{-1}$ центра, рассчитанную с учетом влияния разности диэлектрических проницаемостей ямы и барьеров. Видно, что наведенные заряды сильно увеличивают энергию связи акцепторного

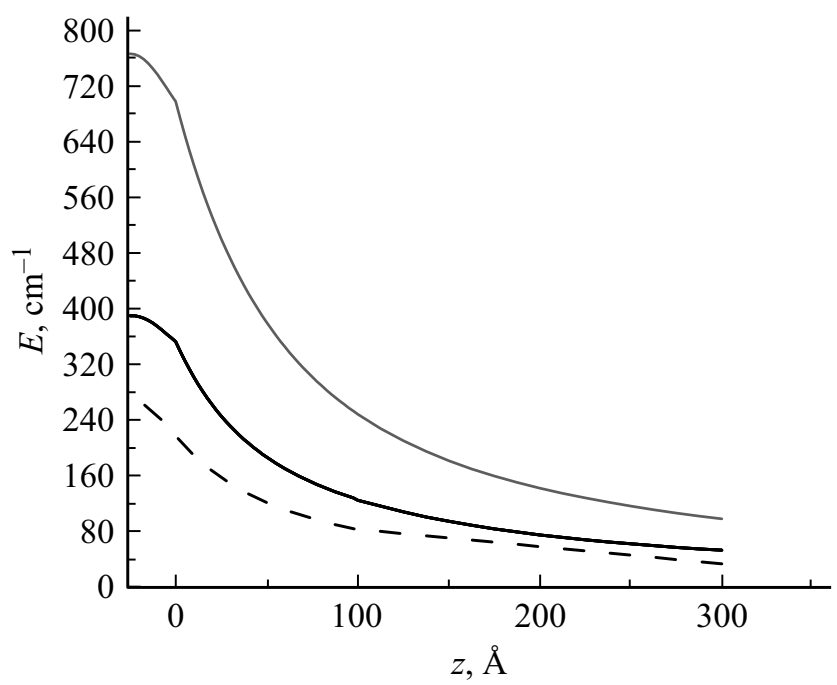

Рис. 3. Рассчитанные зависимости энергии ионизации нейтральной и однократно ионизованной вакансии ртути $\left(A^{0}-\right.$ и $A^{-1}$-центры соответственно) от положения такой вакансии в гетероструктуре $\mathrm{HgTe} / \mathrm{Cd}_{0.7} \mathrm{Hg}_{0.3} \mathrm{Te}$ с КЯ шириной $48 \AA$. Черные линии показывают энергии связи нейтральной вакансии, рассчитанные как без учета влияния наведенных на гетерогранице зарядов (пунктирная линия), так и с учетом влияния зарядов-изображений (сплошная линия). Серая линия показывает энергию связи $A^{-1}$-центра, рассчитанную с учетом влияния разности диэлектрических проницаемостей ямы и барьеров. За начало отсчета $(z=0)$ выбрана гетерограница. центра, расположенного в КЯ или в барьере недалеко от гетерограницы, при этом энергия связи вакансии ртути, помещенной в глубине барьера, меняется меньше.

Из рис. 3 видно, что при смещении акцептора от центра квантовой ямы $(z=-25 \AA$ на графике) к гетерогранице и далее в глубину барьера $(z=300 \AA$ на графике - соответствует толщине барьера [7]) энергия ионизации падает, так энергия связи нейтральной вакансии ртути $A^{0}$-центра меняется от 400 до $50 \mathrm{~cm}^{-1}$, а энергия отрыва второй дырки от $A^{-1}$-центра уменьшается от 760 до $100 \mathrm{~cm}^{-1}$. Следует отметить, что при перемещении дефекта в барьер энергия связи дырки на таком центре не обращается в 0, так как вакансия в барьере создает притягивающий потенциал в КЯ, что приводит к появлению локализованных состояний двумерных дырок в таком потенциале, подобно тому, как описано в работе [11].

При равномерном распределении дефектов по структуре основной вклад в ФЛ будут образовывать оптические переходы с участием состояний центров, расположение которых соответствует участкам плавной зависимости энергии связи от положения акцепторного иона $[11,12]$. Такой участок плавной зависимости энергии ионизации акцепторных центров соответствует дефекту, помещенному в глубину барьера (участок на рис. 3 от $z=100$ до $300 \AA)$. Энергия связи вакансии ртути при этом положении акцептора очень медленно меняется от 130 до $48 \mathrm{~cm}^{-1}$ для $A^{0}$-центра и от 250 до $100 \mathrm{~cm}^{-1}$ для $A^{-1}$ центра. Отметим, что таким энергиям квантов соответствует длинноволновая полоса в спектре ФЛ гетероструктуры $\mathrm{HgTe} / \mathrm{Cd}_{0.7} \mathrm{Hg}_{0.3} \mathrm{Te}$ с КЯ шириной $48 \AA$, исследованной в работе [6].

\section{4. Заключение}

Проведен расчет состояний вакансий ртути в гетероструктурах $\mathrm{HgTe} / \mathrm{CdHgTe} \mathrm{c} \mathrm{квантовыми} \mathrm{ямами,} \mathrm{учиты-}$ вающий существенную разницу значений диэлектрической проницаемости слоев барьеров и квантовых ям. Результаты расчета показали существенное изменение энергии связи акцепторных центров - вакансий ртути из-за наведенного заряда. Так, наведенные заряды сильно увеличивают энергию связи акцепторного центра, расположенного в КЯ или в барьере недалеко от гетерограницы. Энергия связи вакансии ртути, помещенной в глубине барьера, меняется меньше. Рассчитанные энергии ионизации вакансий ртути хорошо согласуются с положением спектральных особенностей в спектре фотолюминесценции гетероструктур $\mathrm{HgTe} / \mathrm{CdHgTe}$ с квантовыми ямами.

\section{Финансирование работы}

Работа выполнена при финансовой поддержке гранта Российского научного фонда № 17-12-01360. 


\section{Конфликт интересов}

Авторы заявляют, что у них нет конфликта интересов.

\section{Список литературы}

[1] I.V. Altukhov, E.G. Chirkova, V.P. Sinis, M.S. Kagan, Yu.P. Gousev, S.G. Thomas, K.L. Wang, M.A. Odnoblyudov, I.N. Yassievich. Appl. Phys. Lett., 79, 3909 (2001).

[2] M.S. Kagan, I.V. Altukhov, V.P. Sinis, S.G. Thomas, K.L. Wang, K.L. Chao, I.N. Yassievich. Thin Sol. Films, 380, 237 (2000).

[3] А.В. Андрианов, А.О. Захарин, Ю.Л. Иванов, М.С. Кипа. Письма ЖЭТФ, 91, 102 (2010).

[4] S.G. Pavlov, H.-W. Hübers, E.E. Orlova, R.Kh. Zhukavin, H. Riemann, H. Nakata, V.N. Shastin. Phys. Status Solidi B, 235, 126 (2003).

[5] V.V. Rumyantsev, D.V. Kozlov, S.V. Morozov, M.A. Fadeev, A.M. Kadykov, F. Teppe, V.S. Varavin, M.V. Yakushev, N.N. Mikhailov, S.A. Dvoretskii, V.I. Gavrilenko. Semicond. Sci. Technol., 32, 095007 (2017).

[6] Д.В. Козлов, В.В. Румянцев, С.В. Морозов, А.М. Кадыков, М.А. Фадеев, М.С. Жолудев, В.С. Варавин, Н.Н. Михайлов, С.А. Дворецкий, В.И. Гавриленко, F. Терре. ЖЭТФ, 154, 1226 (2018).

[7] Д.В. Козлов, В.В. Румянцев, С.В. Морозов, А.М. Кадыков, М.А. Фадеев, В.С. Варавин, Н.Н. Михайлов, С.А. Дворецкий, В.И. Гавриленко, F. Терре. ФТП, 50, 1690 (2016).

[8] S. Fraizzoli, A. Pasquarello. Phys. Rev. B, 42, 5349 (1990).

[9] E.G. Novik, A. Pfeuffer-Jeschke, T. Jungwirth, V. Latussek, C.R. Becker, G. Landwehr. Phys. Rev. B, 72, 035321 (2005).

[10] Г. Бете, Э. Солпите. Квантовая механика атомов $c$ одним и двумя электронами (М., Физматлит, 1960).

[11] В.Я. Алешкин, Б.А. Андреев, В.И. Гавриленко, И.В. Ерофеева, Д.В. Козлов, О.А. Кузнецов. ФТП, 34, 582 (2000).

[12] V.Ya. Aleshkin, B.A. Andreev, V.I. Gavrilenko, I.V. Erofeeva, D.V. Kozlov, O.A. Kuznetsov. Nanotechnology, 11, 348 (2000).

Редактор А.Н. Смирнов

\section{Spectra of double acceptors in barriers and quantum wells layers of the $\mathrm{HgTe} / \mathrm{CdHgTe}$ heterostructures}

\author{
D.V. Kozlov ${ }^{\mathbf{1}, 2}$, V.V. Rumyantsev ${ }^{\mathbf{1 , 2}}$, S.V. Morozov ${ }^{\mathbf{1 , 2}}$ \\ ${ }^{1}$ Institute for Physics of Microstructures \\ Russian Academy of Sciences, \\ 603950 Nizhny Novgorod, Russia \\ ${ }^{2}$ Lobachevsky State University of Nizhny Novgorod, \\ 603950 Nizhny Novgorod, Russia
}

\begin{abstract}
In this paper a theoretical study of the states of double acceptors in $\mathrm{HgTe} / \mathrm{CdHgTe}$ heterostructures with quantum wells, taking into account the significant difference in the values of the dielectric constant of barrier layers and quantum wells was carried out. The effect of such a difference and the charge induced at the heterointerfaces arising from it was described using the potential of charge-images. The calculation showed a significant change in the binding energy of the acceptor centers - mercury vacancies due to the induced charge, and the ionization energies of mercury vacancies are in good agreement with the position of the spectral features in the photoluminescence spectrum of $\mathrm{HgTe} / \mathrm{CdHgTe}$ heterostructures with quantum wells.
\end{abstract}

